\title{
RESPONSABILIDAD PROFESIONAL EN LA ESCUELA NACIONAL DE TRABAJO SOCIAL DE LA UNIVERSIDAD NACIONAL AUTÓNOMA DE MÉXICO
}

\author{
Ulises Torres Sánchez ${ }^{1}$
}

\section{Resumen}

Desde el marco de la responsabilidad profesional, retoma la profesionalización mecanicista, civilizadora e identificante, se elabora el presente reporte de investigación para dar a conocer los principales resultados del estudio realizado a estudiantes de Licenciatura, Especialidad y Maestría en Trabajo Social de la Escuela Nacional de Trabajo Social (ENTS) de la Universidad Nacional Autónoma de México (UNAM). Se revisaron cinco instrumentos de responsabilidad social universitaria en Latinoamérica; Cuestionario de atribución del comportamiento de la Universidad de Concepción, Chile; Medición de la Responsabilidad Social Universitaria de la Universidad Católica de Temuco, Chile; Evaluación de los aspectos de la Responsabilidad Social de la Universidad de Valladolid, España; Cuatro rubros de los impactos educativos de la Universidad León, España y la Construcción Iberoamericana del marco de responsabilidad Social Universitaria de la Universidad de Valencia, España. Este análisis dio como resultado seis fases metodológicas para evaluar este tema en la UNAM, ofrece como resultados el prestigio que posee estudiar en la UNAM, a través de la actividad docente se inserta el concepto de responsabilidad, actividad que se reafirma en las prácticas escolares.

La responsabilidad profesional del egresado se demuestra al momento de interactuar con las personas teniendo en cuenta hacer lo correcto, además de evitar el daño mediante la ética profesional, conocimientos, vocación y empatía con la ciudadanía y la vida digna permite a este profesionista dirigir sus esfuerzos hacia la sociedad. [Palabras clave: ética profesional, responsabilidad social universitaria].

\section{Abstract}

From the context of professional responsibility, takes the mechanistic civilization and professionalization identifier, this research report is made to present the main results of the study of undergraduates, Specialty and Master of Social Work at the National School of Social work (ENTS) of the National Autonomous University of Mexico (UNAM). Five instruments of university social responsibility in Latin America were reviewed; Questionnaire attribution of conduct of the University of Concepción, Chile; Measuring University Social Responsibility of the Catholic University of Temuco, Chile; Evaluating aspects of Social Responsibility of the University of Valladolid, Spain; Four categories of educational impacts of Leon University, Spain and the Iberoamerican University Frame Construction Social responsibility of the University of Valencia, Spain. This analysis resulted in six methodologies to assess this issue in the UNAM phases as a result it provides the prestige that has study at the UNAM, through teaching the concept of responsibility is inserted reaffirms activity in school practices.

Graduate Professional responsibility is demonstrated when interacting with people considering doing the right thing, and avoid the damage by professional ethics, knowledge, dedication and empathy with the public and a dignified life allows this profesionista direct their efforts to society. [Keywords: ethics, university social responsibility].

${ }^{1}$ Profesor de asignatura, Doctor en Ciencias de la Educación, Universidad Autónoma de México. 


\section{Introducción}

\section{Responsabilidad profesional}

La responsabilidad profesional es el compromiso de un egresado con su entorno escolar, laboral, profesional e incluso familiar (Moratalla, 2010, p. 95). Este autor considera que se requiere la mediación "no sólo para resolver conflictos o articular la interdiciplinariedad en la gestión del conocimiento, sino para tender un puente cada vez más necesario entre las instituciones educativas y el fortalecimiento de valores en la sociedad como la justicia y la solidaridad de una nación". También es vital para generar espacios de diálogo social y para despertar la conciencia crítica de las instituciones que gestionan el conocimiento.

\section{Tres modelos de responsabilidad profesional}

Aunque el autor plantea tres modelos, la ausencia de respuesta también representa un modelo que llama naturalista o nihilista. El nihilismo es una opción a la que están apuntando las universidades cuando no se plantean explícitamente la responsabilidad profesional. Los tres modelos son:

Profesionalización mecanicista. La universidad se adapta y ajusta a los mecanismos del mercado laboral. Ante los procesos de modernización se situaría en términos económicos y utilitaristas. Esta opción supone una determinada forma de organizar el conocimiento caracterizado por la separación. Considera que un profesional es un experto, especialista y con determinadas destrezas. La universidad tendría que encargarse de formar en responsabilidad social, forjar el carácter, voluntad de servicio y sentido de justicia.

Profesionalización civilizadora. La universidad no sólo se plantea responder a la modernización económica sino a una modernización cívica. Además de preocuparse por el crecimiento económico o el cumplimiento de las leyes han de estar preocupados por el desarrollo social y el bienestar de la sociedad. La ética de las profesiones supondría una organización del conocimiento donde los saberes se subordinarían a la planificación social y al desarrollo de instituciones de bienestar.

Profesionalización identificante. El profesional tiene la oportunidad de situar su especialización y su capacitación en un amplio conjunto de valores y significados. Los profesionales no sólo son buenos ciudadanos, sino que aspiran a ser personas que tienen un proyecto de vida que comparten, aspiran a una sociedad abierta donde ellos puedan generar valor, desarrollen innovación, creatividad y servicio. La ética profesional facilita la legitimización no sólo económica o jurídica de una profesión sino su credibilidad social, su sentido histórico y su contribución al crecimiento personal en contextos comunitarios del bien común.

El debate sobre la ética de las profesiones es también un debate sobre el sentido y valor de la formación que se imparte en las universidades. La ética de las profesiones desempeña una función mediadora porque tiende un puente entre las tradiciones del conocimiento y las exigencias de la sociedad, entre las diferentes especializaciones y la necesidad de una mínima cultura moral común, entre los intereses pragmáticos de los diferentes grupos sociales y la creación de una ciudadanía crítica, entre la utilidad del trabajo como medio de vida y la actividad profesional como fuente de identidad.

Con este artículo se ubica la responsabilidad profesional de los trabajadores sociales de la Escuela Nacional de Trabajo Social (ENTS) de la Universidad Nacional Autónoma de México (UNAM), en sus tres niveles de enseñanza: licenciatura, especialidad y maestría.

\section{Método}

Se aplicó un cuestionario de 90 reactivos y distribuido en ocho dimensiones. La pregunta eje fue la siguiente: ¿qué responsabilidad adquieren los trabajadores sociales en su formación profesional? Con este trabajo se busca plantear una resignificación del papel de la universidad pública para dar respuesta a las demandas de la sociedad. Como supuesto de trabajo se consideró que "a mayores elementos teóricos, epistemológicos y metodológicos identificados en la formación de conocimientos, habilidades y actitudes de los alumnos de licenciatura, especialidad y maestría de la ENTS UNAM, mayores elementos se tendrán para poder diseñar una propuesta metodológica de evaluación de la responsabilidad profesional". 


\section{Diseño y aplicación del instrumento}

Se llevó a cabo en seis fases.

En la primera, se retomó el análisis de Juan José Martí Noguera (2011, p. 42) sobre la exhaustiva revisión bibliográfica y búsqueda electrónica de redes e instrumentos de responsabilidad, cuyo hallazgo fue la existencia de tres universidades y dos redes en Iberoamérica que cuentan con una estrategia sobre el tema y que diseñaron instrumentos para su evaluación, fundamentados en la docencia, investigación y extensión y difusión de la cultura.

Después de revisar distintas propuestas de responsabilidad (Ortiz, 2009; Universidad de Valladolid, 2009ª Pineda, 1986; Torres, 1997) y se consideraron pertinentes para este análisis cinco de ellas, elaboradas entre 2004 y 2011, con la finalidad de conocer los contenidos propuestos, que dieron origen a la propuesta específica para la ENTS-UNAM (2013).

Se analizaron los cinco instrumentos y se detectaron regularidades significativas dando como resultado los criterios para la construcción del instrumento final (Educar Chile, 2011). El concepto utilizado y los documentos revisados incluyen elementos sobre docencia, investigación y extensión de la cultura y del medio ambiente y compromiso social así como el impacto de la universidad en su entorno; opinión que tienen las personas, alumnos, egresados, docentes y población respecto a los servicios que ofrecen las instituciones de educación superior y la relación de la universidad a partir del prestigio que tiene, su posición como instancia académica, valores éticos adquiridos, compromiso social de los egresados, proyección social de la universidad con los beneficios directos que se ofrecen a partir de actividades culturales, producción editorial, clínicas de servicios, asesorías, consultorías, prácticas profesionales en comunidad, región o instituciones y acciones propias de voluntariado. Las propuestas se relacionan de manera general con la propuesta de Domínguez (2009) que derivaron en la construcción de los reactivos.

La segunda fase consistió en un minucioso análisis de los cinco documentos revisados para el diseño y construcción del instrumento, que se concretó en ocho apartados: datos generales, trabajo social, toma de decisiones, dimensiones, intervención, capital social, ética profesional, la universidad y sus tres funciones sustantivas: docencia, investigación y extensión y difusión de la cultura.

En la tercera fase se diseñaron los criterios para aplicar el instrumento cuya base principal son las tres funciones sustantivas de la UNAM. Se aplicó a los alumnos del último semestre de cada nivel académico de la ENTS que cumplieran con las siguientes características: inscritos en el momento de la aplicación, que formaran parte del sistema escolarizado, regulares (sin adeudo de asignaturas), que informaran de su consentimiento para contestar el cuestionario y que contaran con el conocimiento sobre el plan de estudios para evaluar los temas de las ocho dimensiones predeterminadas.

La cuarta fase se refiere al diseño estadístico de la muestra. El número de alumnos de licenciatura, inscritos en el mes de abril del 2012, era de 386, en las tres especialidades era de 56 y de maestría 44. A partir de estos datos de la población inscrita en la ENTS se programó para un total de 252 estudiantes de los tres niveles académicos. Como no se obtuvo respuesta de 164, se aplicó el cuestionario a 32 hombres y 132 mujeres. Es importante indicar, que la mayor parte de los estudiantes de la ENTS- UNAM son mujeres, por lo que la muestra aplicada tomó en consideración esta situación (ver Tabla 3).

\section{Tabla 1}

Tamaño de la muestra

\begin{tabular}{lcccccc}
\hline Grado & $\begin{array}{c}\text { Total de } \\
\text { estudiantes } \\
\text { inscritos en el } \\
\text { semestre 2012-2 }\end{array}$ & $\begin{array}{c}\text { Población } \\
\text { propuesta a } \\
\text { encuestar }\end{array}$ & $\begin{array}{c}\text { Población } \\
\text { que se } \\
\text { encuestó }\end{array}$ & $\begin{array}{c}\text { Porcentaje } \\
\text { encuestado }\end{array}$ & $\begin{array}{c}\text { Población no } \\
\text { encuestada }\end{array}$ & $\begin{array}{c}\text { Porcentaje } \\
\text { no } \\
\text { encuestado }\end{array}$ \\
\hline Licenciatura & 386 & 167 & $\mathbf{1 1 2}$ & $67 \%$ & 79 & $33 \%$ \\
Especialidad & 56 & 47 & $\mathbf{3 1}$ & $66 \%$ & 25 & $34 \%$ \\
Maestría & 44 & 38 & $\mathbf{2 1}$ & $55 \%$ & 17 & $45 \%$ \\
Total & 486 & 252 & $\mathbf{1 6 4}$ & $\mathbf{6 5 \%}$ & $\mathbf{1 2 1}$ & $\mathbf{4 5 \%}$ \\
\hline
\end{tabular}

Fuente: UNAM-ENTS (2012) Sistema de Administración Escolar. Informe estadístico 2012. Secretaría de Apoyo y Desarrollo Escolar. México. UNAM. 
Con este trabajo se buscaba plantear una resignificación del papel de la universidad pública para dar respuesta a las demandas de la sociedad. Como supuesto de trabajo se consideró que a mayores elementos teóricos, epistemológicos y metodológicos identificados en la formación de conocimientos, habilidades y actitudes de los alumnos de licenciatura, especialidad y maestría de la ENTS UNAM, mayores elementos se tendrán para poder diseñar una propuesta metodológica de evaluación de la responsabilidad profesional.

En la quinta y última fase, se planteó la estrategia de recolección de la información. Se localizaron a los alumnos de los tres niveles: licenciatura, tres especialidades y la maestría, del último semestre, en 2012, directamente en sus salones de clase, con la autorización previa de sus profesores. Se aceptó la decisión de los alumnos que no quisieron contestar el instrumento: 75 de licenciatura, 25 de especialidad y 17 de maestría. La actividad se distribuyó en ambos turnos de (matutino y vespertino).

Se requirió de un equipo de ocho encuestadores quienes recolectaron la información con alumnos de los tres niveles, en un lapso de 96 horas en promedio. Se capturó la información a lo largo de 60 horas durante dos semanas, que comprendió del 21 de mayo al 1 de junio de 2012.

Tablas de resultados.

\section{Tabla 2}

\section{Grupo Edad}

Grupo Edad Nivel académico que cursa actualmente

Total

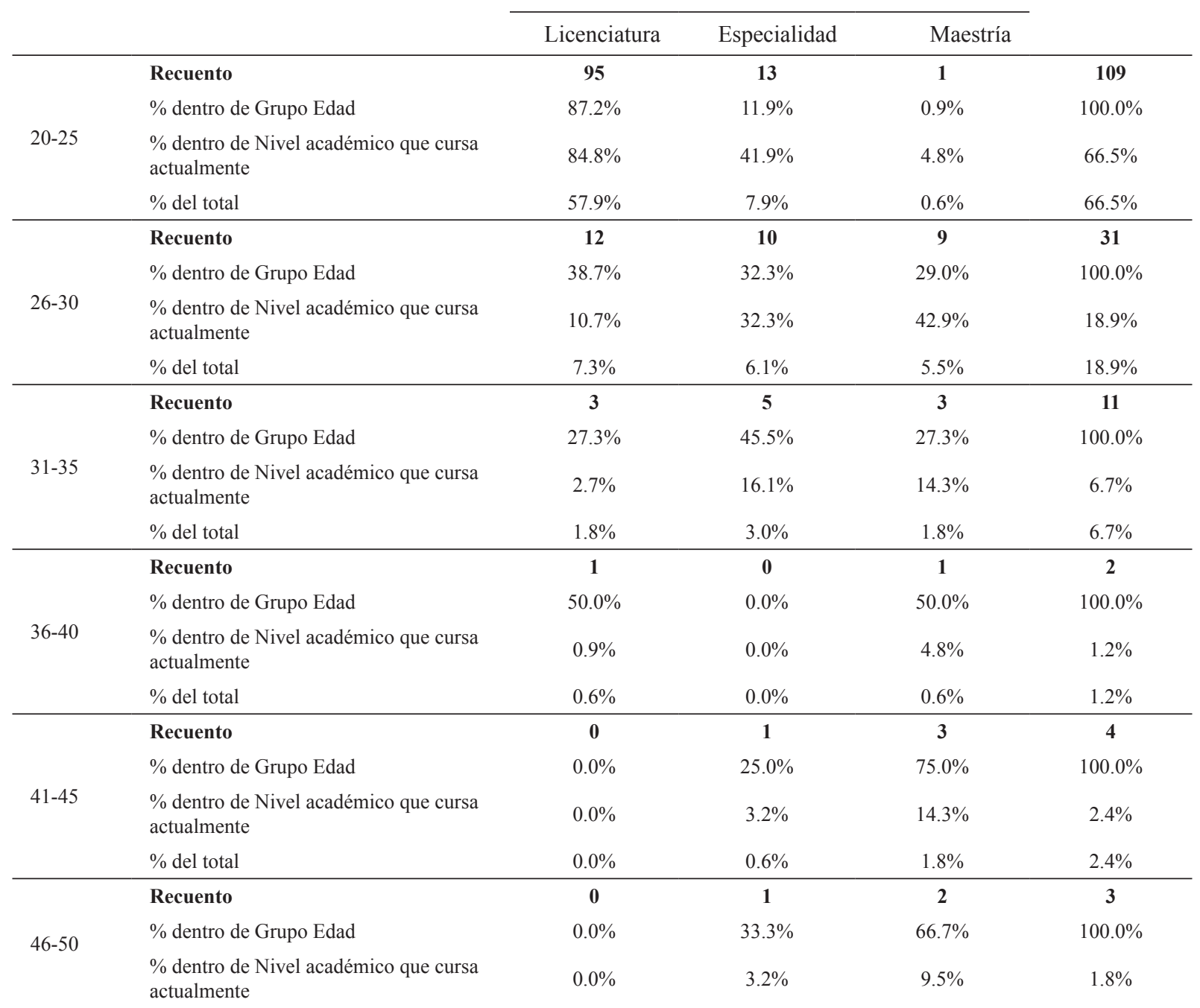




\section{Tabla 2}

Grupo Edad

\begin{tabular}{|c|c|c|c|c|c|}
\hline \multicolumn{2}{|c|}{ Grupo Edad } & \multicolumn{3}{|c|}{ Nivel académico que cursa actualmente } & \multirow{2}{*}{ Total } \\
\hline & & Licenciatura & Especialidad & Maestría & \\
\hline \multirow[t]{3}{*}{$51-55$} & Recuento & 1 & 1 & 2 & 4 \\
\hline & $\%$ dentro de Grupo Edad & $25.0 \%$ & $33.3 \%$ & $66.7 \%$ & $100.0 \%$ \\
\hline & $\begin{array}{l}\% \text { dentro de Nivel académico que cursa } \\
\text { actualmente }\end{array}$ & $0.9 \%$ & $3.2 \%$ & $9.5 \%$ & $1.8 \%$ \\
\hline \multirow[t]{3}{*}{ Total } & Recuento & 112 & 31 & 21 & 164 \\
\hline & $\%$ dentro de Grupo Edad & $100.0 \%$ & $100.0 \%$ & $100.00 \%$ & $100.0 \%$ \\
\hline & $\begin{array}{l}\% \text { dentro de Nivel académico que cursa } \\
\text { actualmente }\end{array}$ & $68.29 \%$ & $18.9 \%$ & $12.80 \%$ & $100.0 \%$ \\
\hline
\end{tabular}

Los alumnos más jóvenes se concentran en el nivel de licenciatura $57.9 \%$ de 20 a 25 años, en el rango de 26 a 30 años $7.3 \%$, de 31 a 35 años 1.8\%. En las tres especialidades, predomina el rango de 20 a 25 años donde se concentra el 7.9\%. En la maestría, 5.5\% pertenece al grupo de edad de 20 a 25 años, personas jóvenes preparándose en los tres niveles académicos.

Aunque en los niveles de especialidad y maestría estudian personas extranjeras. Por ejemplo, americanas, colombianas, chilenas, y venezolanas entre otras nacionalidades, los estudiantes de intercambio académico optaron por no responder el instrumento reduciéndose así la muestra a los alumnos de nacionalidad mexicana. La mayor concentración de la muestra es $68.3 \%$ de licenciatura, seguido del $18.9 \%$ en las especialidades y $12.8 \%$ inscritos en el nivel académico de la maestría.

Una variable determinante en la muestra fue el nivel académico que cursan actualmente los estudiantes que componen el universo al que se aplicó el cuestionario. Por ese motivo las tablas se presentan principalmente separados por nivel académico.

En licenciatura, $42.9 \%$ eligió la carrera porque le agrada el trabajo social, ya que contribuye al desarrollo de las personas que atiende, $25.9 \%$ por las asignaturas que contempla el plan de estudios, $22.3 \%$ la eligió como segunda opción pues no está convencido del motivo por el cual la estudia y $8.9 \%$ considera que es multidisciplinaria.

En especialidad, 48.4\% la eligió porque le agrada el trabajo social y contribuye al desarrollo de las personas, $32.3 \%$ por las asignaturas que integran el plan de estudios, $16.1 \%$ la eligió como segunda opción y $3.2 \%$ la considera multidisciplinaria.

En la maestría, $47.6 \%$ la eligió porque le agrada el trabajo social y contribuye al desarrollo de las personas, $28.6 \%$ por las asignaturas que integran el plan de estudios y $9.5 \%$ fue su segunda opción.

Del total, $44.5 \%$ la eligió porque contribuye al desarrollo de las personas, $27.4 \%$ por las asignaturas del plan de estudios, para el $19.5 \%$ fue su segunda opción y $8.5 \%$ la considera multidisciplinaria.

Los principales motivos por los cuales decidieron estudiar en la UNAM son: a nivel de licenciatura, $66.1 \%$ por el prestigio que tiene estudiar dentro de la Universidad Nacional Autónoma de México, 23.2\% porque consideran que es la única escuela que imparte la carrera de trabajo social dentro de la UNAM, el $7.1 \%$ porque realizó su pase automático a la UNAM y el $3.6 \%$ por las materias contempladas en el plan de estudios.

Un $61.3 \%$ de alumnos en especialidad la eligió por el prestigio de estudiar en la UNAM, 25.8\% porque es la única que la imparte en la UNAM y DF. 9.7\% por pase automático y $3.2 \%$ por las materias que contempla el plan de estudios.

En maestría, $57.1 \%$ por el prestigio, $23.8 \%$ por el plan de estudios, $14.3 \%$ por el pase automático y $4.8 \%$ porque es la única escuela que imparte la carrera en la UNAM. 


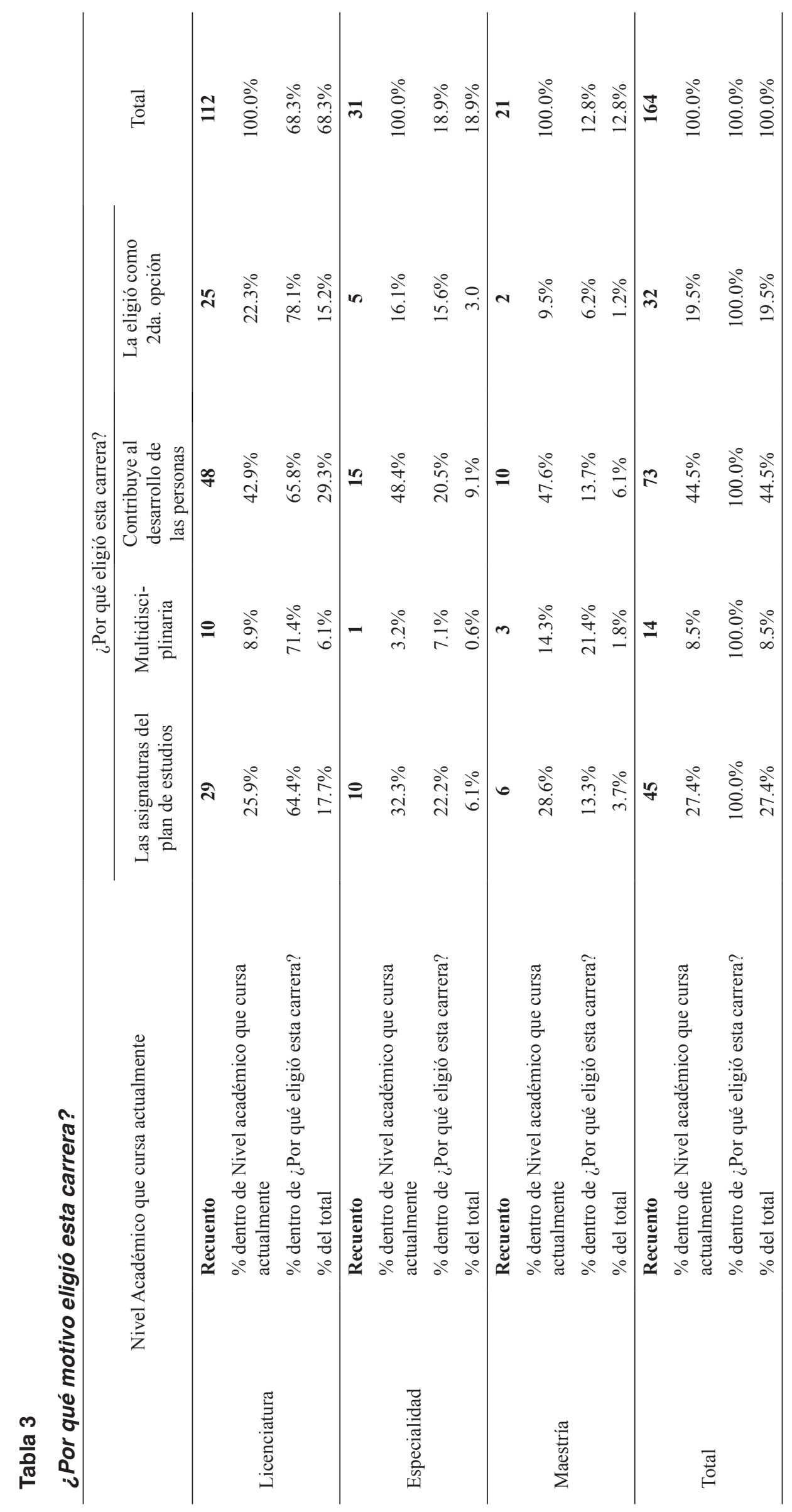




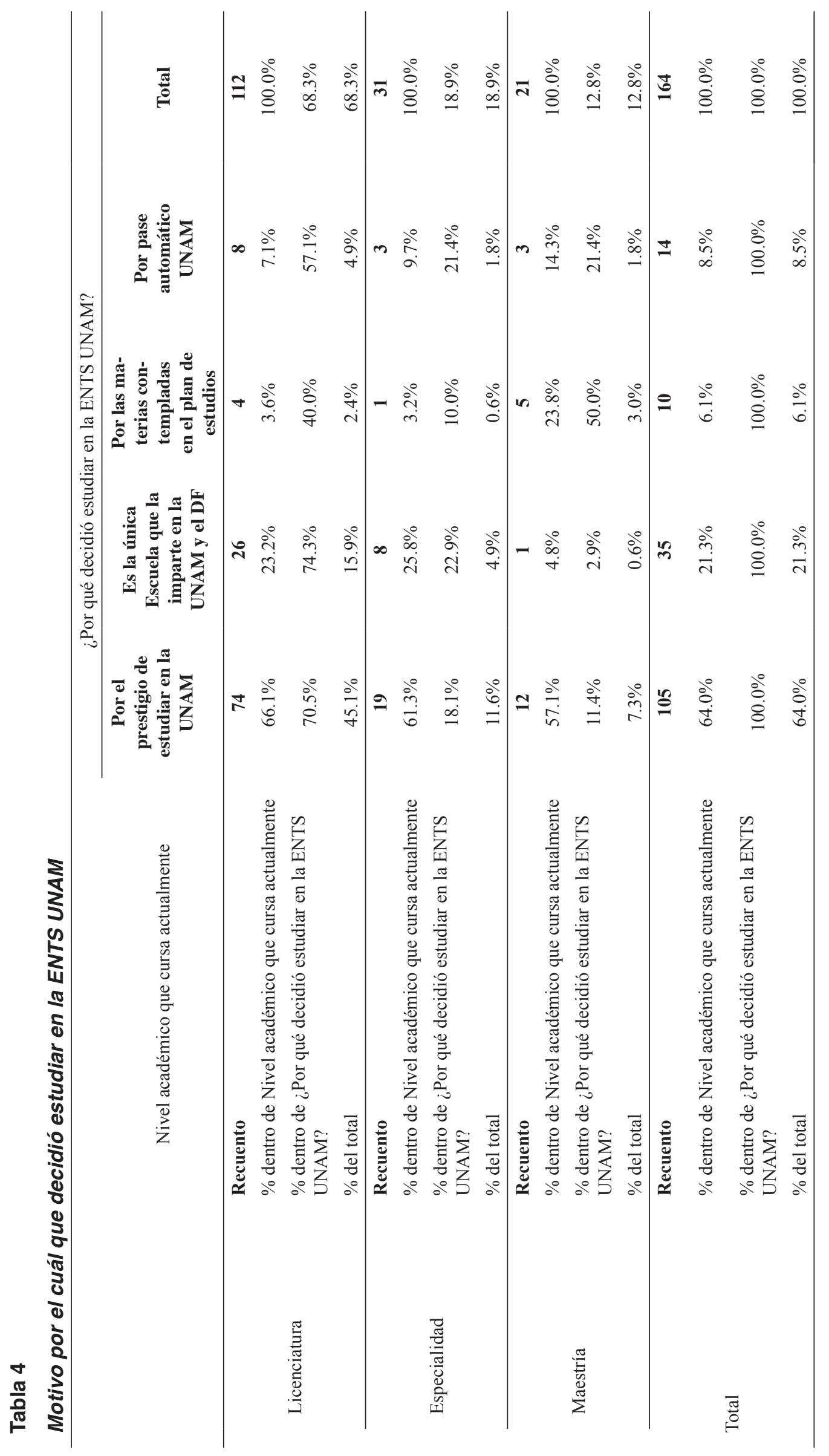




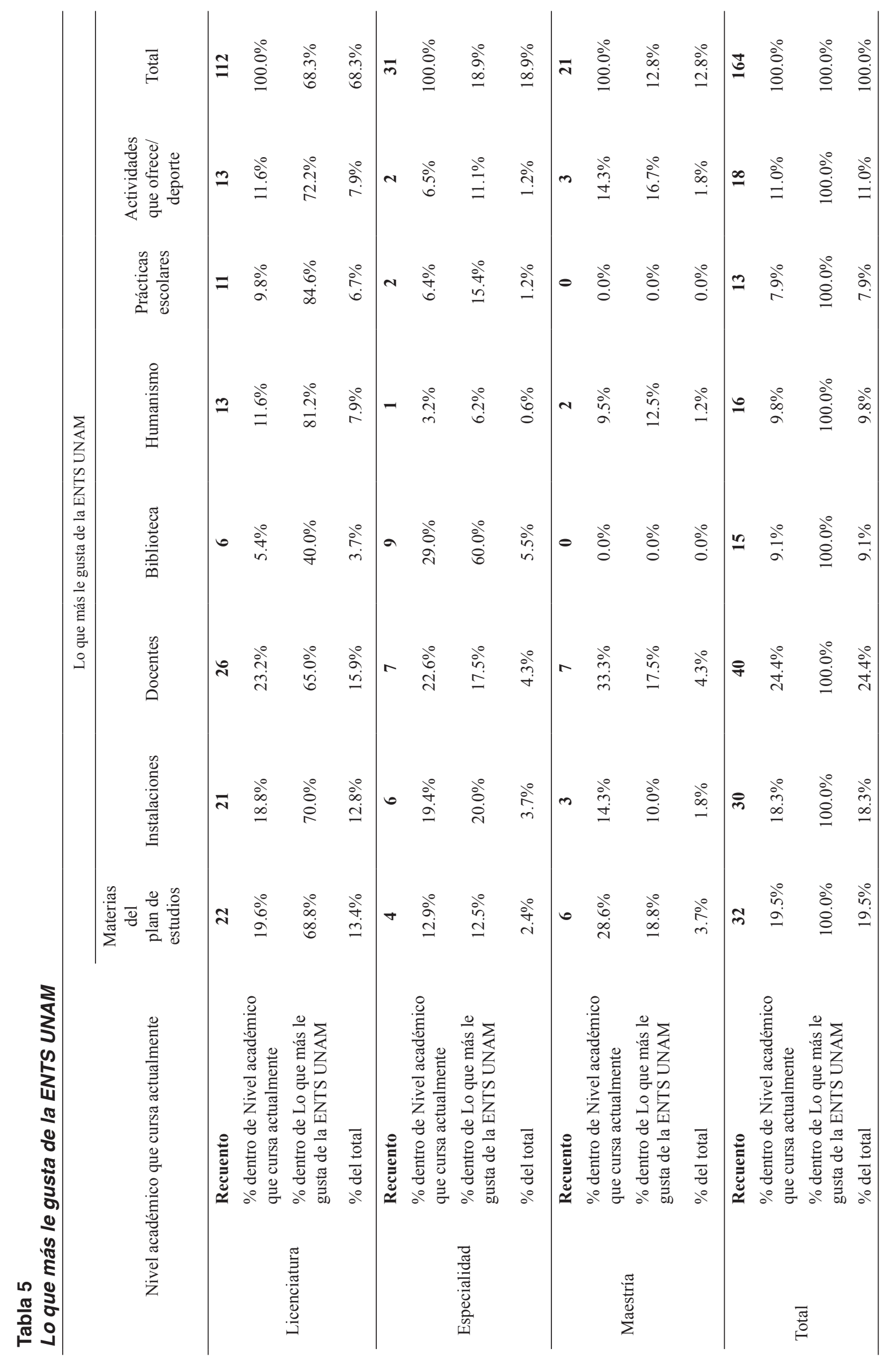


Se presentan los resultados del más alto al más bajo por nivel académico; lo que más les gusta de la ENTS UNAM a los estudiantes de la licenciatura es la capacidad y preparación que presentan los docentes $23.2 \%$, materias del plan de estudios $19.6 \%$, infraestructura física que presentan las instalaciones $18.8 \%$, humanismo en la carrera $11.6 \%$ y actividades que ofrece como el deporte $11.6 \%$, aprendizaje que se obtiene dentro de las practicas escolares $9.8 \%$ y el acervo que contiene la biblioteca $5.4 \%$.

En especialidad $29 \%$ considera que los contenidos que tiene la biblioteca para realizar la investigación son trascendentes, la formación profesional que tienen los docentes $22.6 \%$, instalaciones y centros de cómputo para el desarrollo de la investigación de tesis $19.4 \%$, materias del plan de estudios $12.9 \%$, deporte $6.5 \%$, prácticas, $6.4 \%$, y el humanismo de la maestría con $3.2 \%$.

Dentro de la maestría la opinión de 33.3\% establece que es la elevada preparación académica de los docentes, materias del plan de estudios ayudan a resolver de mejor manera problemas y necesidades sociales que se presentan en su ejercicio profesional $28.6 \%$, actividades que ofrece $14.3 \%$, instalaciones $14.3 \%$ y humanismo $9.5 \%$.

Los licenciados y maestros opinan que los docentes son los más importantes. Para los especialistas y maestrantes es la biblioteca. De manera inversa lo menos importante para los licenciados es el acervo de la biblioteca debido a que no saben utilizar, buscar, seleccionar, discernir, leer, analizar y resumir la densa información que se presenta. Los especialistas no consideran al humanismo como un elemento trascendente pues saben que se aplica al momento de intervenir y para los maestrantes son las prácticas y la biblioteca pues ellos ya se encuentran ejerciendo la profesión en un espacio directo donde aplican los conocimientos, habilidades y actitudes que han aprendido en su formación profesional.

A nivel de licenciatura, al $45.5 \%$ les gusta la multidisciplina del plan de estudios ya que pueden insertarse en distintas áreas de ejercicio profesional, $21.4 \%$ considera que el aprendizaje en la práctica comunitaria, regional y de especialización les ofrecen la posibilidad de aplicar lo aprendido en clase y contrastar la teoría con la realidad social, $14.3 \%$ establece que los campos de acción profesional donde pueden desarrollarse son interesantes, $9.8 \%$ la intervención con casos grupos y comunidad, $4.5 \%$ la investigación y $4.5 \%$ la calidad de formación profesional de los docentes.

En la especialidad, 54.8\% considera a la multidisciplina como lo que más le gusta, $19.4 \%$ prácticas escolares les brinda la posibilidad de aprendizaje, $12.9 \%$ la posibilidad de poderse incorporar al mercado de trabajo en distintos campos de intervención profesional, $12.9 \%$ la metodología de casos, grupos y comunidades que se establece en las distintas instituciones para poder intervenir con la población.

En la maestría, $47.6 \%$ afirma que la multidisciplina del plan de estudios, $23.8 \%$ se inclina por la metodología del trabajo social a nivel de casos, grupos y comunidad para intervenir, $9.5 \%$ los campos de intervención donde pueden trabajar, $9.5 \%$ la practica en sus distintos niveles, $4.8 \%$ la investigación que se realiza y $4.8 \%$ la calidad académica de los docentes.

En los tres niveles académicos se observa que lo más interesante es la multidisciplina del plan de estudios lo que les permite entender de manera general, holística y completa la realidad social que se les presenta en una situación real. En sentido contrario, lo que menos agrada es la investigación y la calidad de los docentes, acciones que deberán reforzarse si es que se pretende alinear los planes y programas de estudio bajo los conceptos de la RSU en la ENTS-UNAM.

En este punto no se cubren dos importantes aspectos de las funciones sustantivas de la UNAM: la investigación y la docencia. Se requiere un diagnóstico general de las necesidades de los estudiantes para poder apuntalar una mejor selección de docentes e investigación que trascienda para poder cumplir con dichas funciones. Producción bibliográfica en el contexto mexicano y además el respeto por el medio ambiente, con estos elementos la ENTS se acercará más a convertirse en una Facultad Socialmente Responsable. 


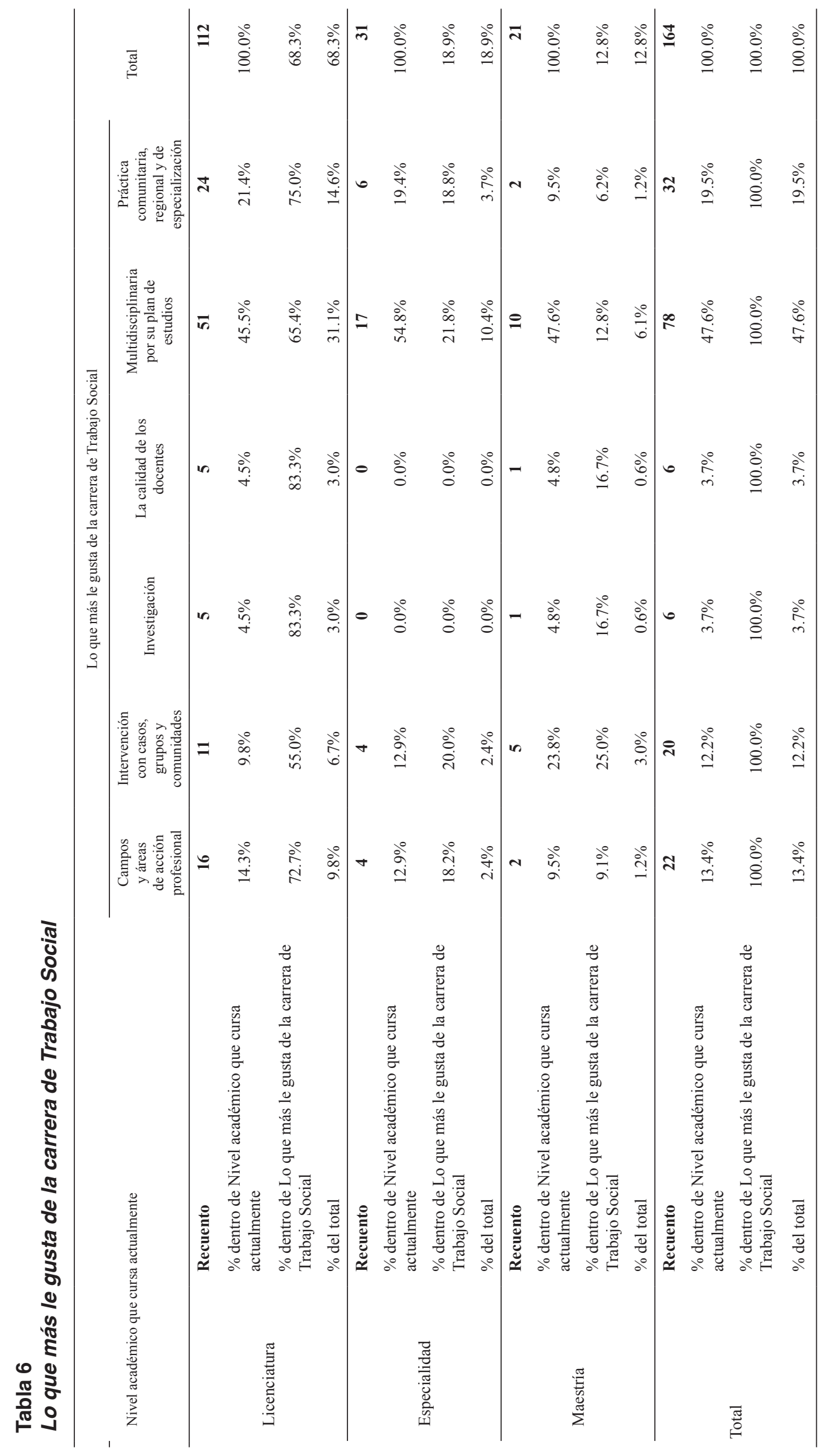




\section{Tabla 7}

\section{Por qué eligió la carrera de trabajo social como primera opción}

\begin{tabular}{|c|c|c|c|c|}
\hline \multicolumn{2}{|c|}{ Nivel académico que cursa actualmente } & \multicolumn{2}{|c|}{$\begin{array}{c}\begin{array}{c}\text { Eligió la carrera de trabajo social como primera } \\
\text { opción }\end{array}\end{array}$} & \multirow{2}{*}{ Total } \\
\hline \multirow[b]{3}{*}{$\cdot \cdot$} & & $\mathrm{Si}$ & No & \\
\hline & Recuento & 77 & 35 & 112 \\
\hline & $\begin{array}{l}\% \text { dentro de Nivel académico que cursa } \\
\text { actualmente }\end{array}$ & $68.8 \%$ & $31.2 \%$ & $100.0 \%$ \\
\hline \multirow{2}{*}{ Licenciatura } & $\begin{array}{l}\text { \% dentro de Eligió la carrera de trabajo } \\
\text { social como primera opción }\end{array}$ & $72.6 \%$ & $60.3 \%$ & $68.3 \%$ \\
\hline & $\%$ del total & $47.0 \%$ & $21.3 \%$ & $68.3 \%$ \\
\hline \multirow{4}{*}{ Especialidad } & Recuento & 19 & 12 & 31 \\
\hline & $\begin{array}{l}\% \text { dentro de Nivel académico que cursa } \\
\text { actualmente }\end{array}$ & $61.3 \%$ & $38.7 \%$ & $100.0 \%$ \\
\hline & $\begin{array}{l}\text { \% dentro de Eligió la carrera de trabajo } \\
\text { social como primera opción }\end{array}$ & $17.9 \%$ & $20.7 \%$ & $18.9 \%$ \\
\hline & $\%$ del total & $11.6 \%$ & $7.3 \%$ & $18.9 \%$ \\
\hline \multirow{4}{*}{ Maestría } & Recuento & 10 & 11 & 21 \\
\hline & $\begin{array}{l}\% \text { dentro de Nivel académico que cursa } \\
\text { actualmente }\end{array}$ & $47.6 \%$ & $52.4 \%$ & $100.0 \%$ \\
\hline & $\begin{array}{l}\text { \% dentro de Eligió la carrera de trabajo } \\
\text { social como primera opción }\end{array}$ & $9.4 \%$ & $19.0 \%$ & $12.8 \%$ \\
\hline & $\%$ del total & $6.1 \%$ & $6.7 \%$ & $12.8 \%$ \\
\hline \multirow{4}{*}{ Total } & Recuento & 106 & 58 & 164 \\
\hline & $\begin{array}{l}\% \text { dentro de Nivel académico que cursa } \\
\text { actualmente }\end{array}$ & $64.6 \%$ & $35.4 \%$ & $100.0 \%$ \\
\hline & $\begin{array}{l}\% \text { dentro de Eligió la carrera de trabajo } \\
\text { social como primera opción }\end{array}$ & $100.0 \%$ & $100.0 \%$ & $100.0 \%$ \\
\hline & $\%$ del total & $64.6 \%$ & $35.4 \%$ & $100.0 \%$ \\
\hline
\end{tabular}

Dentro de la licenciatura, $68.8 \%$, de la especialidad $61.3 \%$ y de la maestría $47.6 \%$ eligieron la carrera de trabajo social, mientras que $31.2 \%$ de la licenciatura, $38.7 \%$ de la especialidad y $52.4 \%$ de la maestría no la eligieron como primera opción. Seis de cada diez eligen la carrera, cuatro de cada diez no la eligen.

Entre las principales características académicas encontradas en los estudiantes que no eligieron la carrera, es que hicieron su preparatoria o bachillerato mayor a tres años con promedio inferior a 8 , lo que provoca que la UNAM los envíe a otra carrera que ellos no habían elegido, debido a los resultados presentados no garantiza que concluyan. Surge aquí una pregunta de investigación ¿qué identidad profesional desarrollan los alumnos que no eligieron la carrera como primera opción?

El $64.6 \%$ del total de la muestra eligió la carrera de trabajo social como primera opción, mientras que el $35.4 \%$ no la eligió. Supone entonces que no necesariamente desarrollan el compromiso social, identidad profesional, valores éticos o derechos humanos. En comparación con los que eligieron la carrera como primera opción desarrollan un mayor número de características en comparación con aquellos que no la eligieron como primera opción.

Como se explicará más adelante, se abre una nueva pregunta de investigación posterior a esta publicación: ¿qué conocimientos, habilidades, destrezas y aptitudes adquiere un alumno que no eligió la carrera?, ¿qué tan significativa es la formación de una persona que no quería la carrera como primera opción pero qué sin embargo concluye?, ¿cuál es el seguimiento que se le brinda a este profesionista? y ¿cómo intervienen profesionalmente los egresados que no eligieron la carrera en comparación con los que si eligieron la carrera? Preguntas que se resolverán en futuras indagaciones científicas con estudios de seguimiento de egresados, estudios de empleadores y a usuarios. 


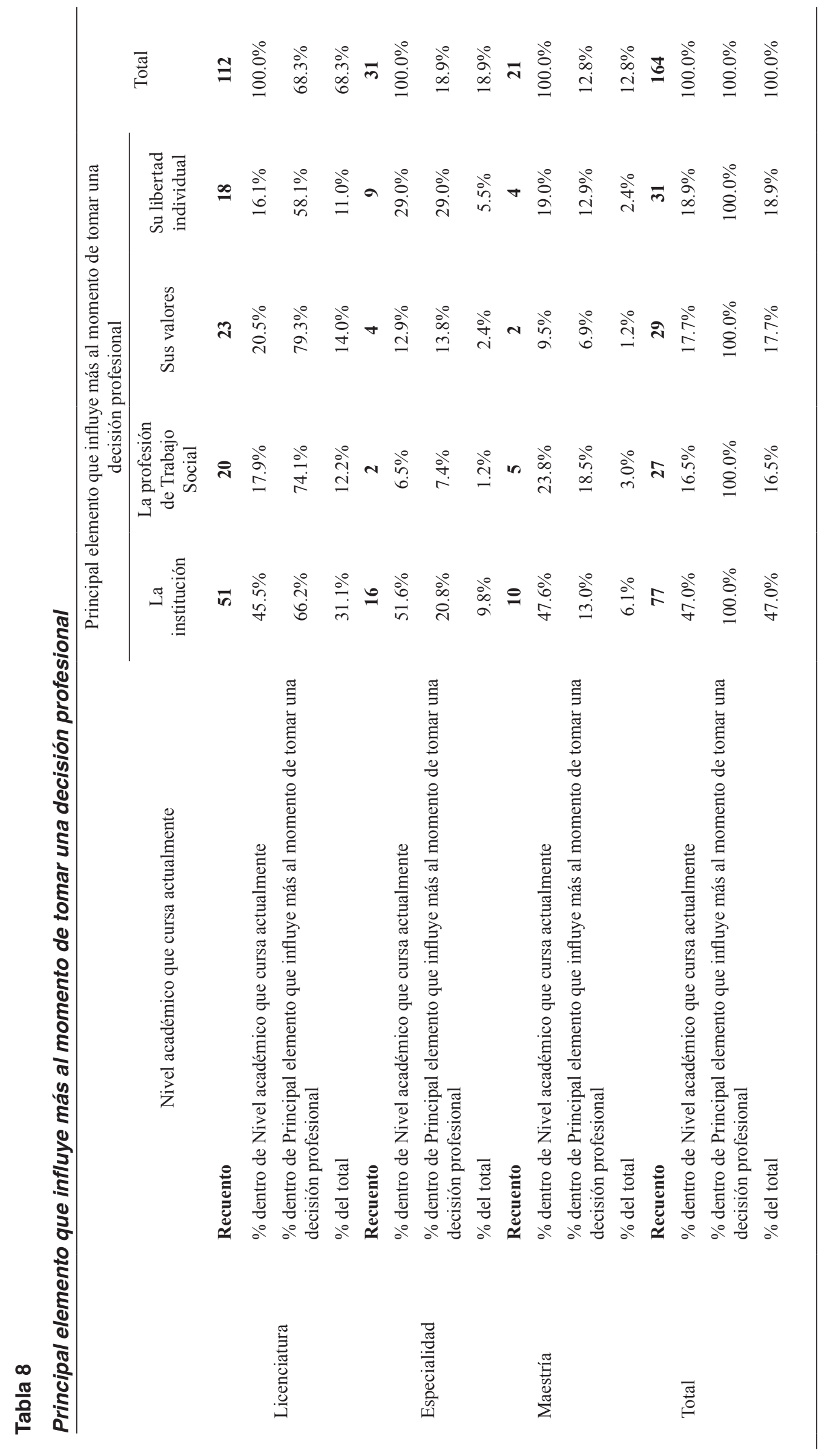




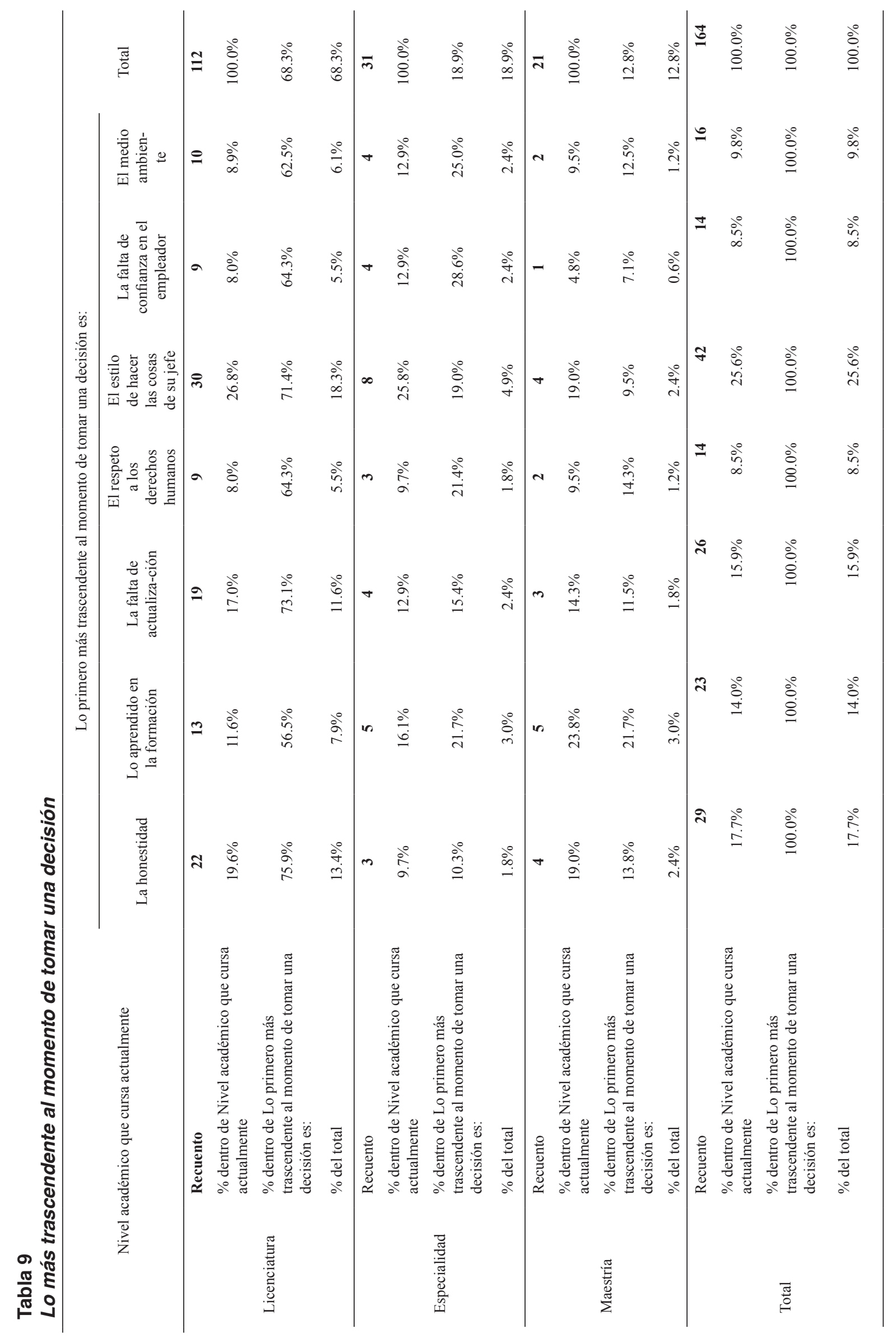


En licenciatura, 45.5\% establece que la institución es quien determina el tipo de intervención profesional para el trabajador social, el $20.5 \%$ son sus valores, el $17.9 \%$ los postulados generales de la disciplina del trabajo social y $16.1 \%$ lo determina su libertad individual al momento de intervenir con una persona. En especialidad, 51.6\% dice que la institución determina su intervención profesional, 29\% la libertad individual, $12.9 \%$ los valores y $6.5 \%$ la profesión del trabajo social.

En maestría, $47.6 \%$ lo determina la institución, 23.8\% la profesión de trabajo social, 19\% su libertad individual, $9.5 \%$ sus valores. Los tres niveles académicos coinciden en que la institución determina la intervención profesional del trabajador social, pues le permite o limita el tipo y profundidad de esa intervención, de acuerdo con los manuales de procedimientos, estrategias y modelos teóricos establecidos por la institución y de aquello que se espera sean utilizados como instrumentos y herramientas del trabajo social.

El componente que más influye al momento de tomar una decisión profesional en licenciatura es el estilo de hacer las cosas de su jefe o profesor $26.8 \%$, honestidad $19.6 \%$, falta de actualización $17 \%$, lo aprendido en la formación $11.6 \%$, el medio ambiente $8.9 \%$, respeto a los derechos humanos $8 \%$ y falta de confianza del empleador hacia el trabajador social $8 \%$.

En la especialidad, el estilo de hacer las cosas de su jefe $25.8 \%$, lo aprendido en la formación $16.1 \%$, falta de actualización $12.9 \%$, falta de confianza del empleador $12.9 \%$, medio ambiente $12.9 \%$ y el respeto a los derechos humanos $9.7 \%$. En la maestría lo aprendido en la formación $23.8 \%$, el estilo de hacer las cosas de su jefe $19 \%$, honestidad 19\%, falta de actualización $14.3 \%$, medio ambiente $9.5 \%$, respeto a los derechos humanos $9.5 \%$ y falta de confianza en el empleador $4.8 \%$.

Si las personas y los jefes que tienen a su cargo a profesionales de trabajo social contaran con experiencia en la impartición de clases, podrían enseñar a los futuros profesionistas la forma de realizar la intervención profesional. Con esta acción un mayor número de empleadores podría contratar a más trabajadores sociales, otorgando confianza en su desempeño laboral. La experiencia profesional sería aplicada al interior de los temas que se imparten y a su vez, el curriculum se relacionaría mucho más con las necesidades que requiere el mercado de trabajo.

\section{Aportes de la investigación}

Entre los principales hallazgos encontrados están los elementos que reúnen un modelo de RSU que incluye cinco puntos: gestión, docencia, investigación, extensión y difusión de la cultura además de la consideración sobre el medio ambiente. El enfoque teórico de la responsabilidad se fundamenta en cinco aspectos; laboral, económico, ambiental, ético y social de las instituciones de educación superior. La responsabilidad está presente cuando se toma conciencia del ejercicio profesional, de la sociedad a la que beneficia y la protección que se brinda al medio ambiente. Estas características responden a las tres funciones sustantivas de la universidad: docencia, investigación y difusión, y extensión de la cultura.

Se elaboró la metodología para el diseño, aplicación y evaluación de las dimensiones de la responsabilidad para la ENTS-UNAM que puede ser de utilidad para las otras escuelas de trabajo social en México y para otras investigaciones sobre esta temática. Se ubicaron elementos significativos que se relacionan con dicho campo temático.

La UNAM forma implícitamente en materia de responsabilidad profesional a sus estudiantes por medio de la formación en algunos valores y actitudes y en elementos acerca de la ética profesional y de los derechos humanos.

El profesionista universitario recibe una educación, Profesionalización mecanicista, ya que se ajusta al mercado laboral observando los procesos de modernización que forja su carácter, voluntad de servicio y sentido de justicia. Aporta una Profesionalización civilizadora a través de su formación en valores y por último, obtiene una Profesionalización identificante ya que sitúa al estudiante como un ciudadano abierto, que genera valor agregado, innovador, creativo y con vocación de servicio para el país.

Con este artículo se ubica la responsabilidad profesional de los trabajadores sociales de la Escuela Nacional de Trabajo Social (ENTS) de la Universidad Nacional Autónoma de México (UNAM), en 
Sus tres niveles de enseñanza: licenciatura, especialidades y maestría.

\section{Referencias}

Asociación Internacional de Escuelas de Trabajo Social. (2012). http://trabajadorasocialenlared. blogs pot. $\mathrm{mx} / 2010 / 06 / \mathrm{la}-\mathrm{asociacion}$ internacional-de-escuelas.html

Bermejo, Francisco. (2002). Ética y Trabajo Social. España: Publicaciones de la Pontificia Universidad de Comillas.

Domínguez, María. (2009). Cuatro rubros: impacto de funcionamiento organizacional, impactos educativos, impactos cognoscitivos $y$ epistemológicos e impactos sociales. Cuestionario. España: Universidad de León.

Educar Chile. (2011). Tabla de especificaciones de una prueba para tus clases. Evaluación. Objetivos, contenidos, tipos, cantidades, pesos de las preguntas. ¿Cómo construir, probar, ajustar y afinar un instrumento? Ministerio de educación. Chile. Recuperado de: http:// www.educarchile.cl/elportal.herramientas/ planificacion1610/article-96393.html. Autor

Federación Internacional del Trabajo Social. (1990). Propuesta de declaración sobre los principios éticos en Trabajo Social, Argentina. Argentina.

Martí, Juan. (2011). Responsabilidad SocialUniversitaria: estudio acerca de los comportamientos, los valores y la empatía en estudiantes de universidades Iberoamericanas. Trabajo de grado, Doctorado en Psicología Básica Universitat de Valéncia Estudi General, España.

Moratalla, Domingo. (2010). Ética de las profesiones y formación universitaria: tres modelos de responsabilidad profesional. La riqueza ética de las profesiones, (12) 91 - 109. Ortiz, Iván. (2009). Proyección y Responsabilidad Social en las Facultades de Derecho. Derecho PUCP. Pontifica Universidad Católica del Perú. Perú. (Pág. 263-271). Capítulo 8. En Educación Superior y Sociedad. Nueva Época. Año. 13,

Pineda, Rafael. (1986). Responsabilidad de la educación superior frente a las demandas del desarrollo del país. Trabajo de grado. Doctorado. Universidad Rafael Landivar CINDEG). Guatemala.

Presidencia de la República. (2002). Compromiso Social por la Educación. México: Gobierno Federal.

Torres, Ulises. (2013) Construcción de un instrumento para conocer la responsabilidad social universitaria de la Escuela Nacional de Trabajo Social de la Universidad Nacional Autónoma de México. Trabajo de grado. Doctorado. Colegio de Estudios de Posgrado de la Ciudad de México A.C. México.

Torres, Manuel. (1997). La responsabilidad social universitaria. Tesis Doctoral. Centro de investigaciones y desarrollo de la Educación. Guatemala: Universidad Rafael Ladivar.
Universidad Construye País (2006). Una manera de ser Universidad, teoría y práctica en la experiencia chilena. Edición proyecto Universidad construye país. Santiago de Chile.

Universidad de Valladolid (2009a). Evaluación del grado en que se incluyen contenidos 0 perspectivas relacionados con aspectos de la responsabilidad social. Informe de resultados sobre la presencia de contenidos de responsabilidad social en la formación universitaria de Valladolid España (Uva). España.

Universidad de Valladolid. (2009b). Factoría de Responsabilidad Social. Revista Memorias Caja de Burgos. España.

Vallaeys, Francois. (2009). Responsabilidad Social Universitaria: Una nueva filosofía de gestión ética e inteligente. UNESCO.

Vázquez, Octavio. (2004). Libro Blanco. Título de Grado en Trabajo Social. España: Agencia Nacional de Calidad y Acreditación. Universidad de Huelva. 